Vietnam Journal of Mechanics, VAST, Vol.34, No. 1 (2012), pp. $45-54$

\title{
THREE-AXIS PIEZORESISTIVE ACCELEROMETER WITH ADJUSTABLE AXIAL RESOLUTIONS
}

\author{
Bui Thu Hang, Tran Duc Tan, Chu Duc Trinh \\ University of Engineering and Technology, $V N U$
}

\begin{abstract}
A three-axis piezoresistive accelerometer which has adjustable resolutions to three axes was developed using MicroElectroMechanical Systems (MEMS) technology. This sensor made of a heavy proof mass and four long beams is to obtain high resolutions by reducing resonance frequencies. Adjustable resolution with small cross axis sensitivity could be obtained by a three-dimensional sensor structure.
\end{abstract}

Keywords: MicroElectroMechanical Systems (MEMS), accelerometer, piezoresistance.

\section{INTRODUCTION}

Micro-machined inertial sensors that consist of accelerometers and gyroscopes have a significant percentage of silicon based sensors. Accelerometers have the second largest sale volume after pressure sensors [1] and can be found mainly in automotive industry, biomedical applications, household electronics, robotics, vibration analysis, navigation systems, and so on. Variety of accelerometers has increased based on different principles such as capacitive, piezoresistive, piezoelectric. The concept of accelerometer is not new, but the demand from commerce has motivated continuous research in order to minimize the size and improve its performance.

The realistic applications create a huge motivation for the widely research of MEMS based sensors, especially accelerometers. In the modern world, applications require new sensors with smaller size and higher performance. However, there are few results which can bring out efficient and comprehensive methodologies for accelerometer designs in practice.

\section{LITERATURE SURVEY AND LIMITATIONS OF EXISTING METHODS}

In fact, there is a lack of research which focuses on optimizing the performance of the multi-axis accelerometer.

In 1997, Ramos J. [2] presented a lateral capacitive structure that could enhance the sensitivity by width optimization. An optimum assignment is thus found for the distribution of area in surface micro-machined lateral capacitive accelerometers between stationary and movement of the sensor.

In 2000, Harkey J.A. et al. [3] presented flicker noise considerations for the design and process optimization of piezoresistive cantilevers. In this work, data was shown to 
validate the Hooge model for the flicker noise in piezoresistive cantilevers. Following the equations of the Hooge and Johnson noise, and sensitivity, an expression was derived to predict the force resolution of a piezoresistive cantilever based on its geometry and processing. Using this expression, an optimization analysis was also performed.

In 2004, Sankar A.R. et al. [4] presented temperature drift analysis of the silicon micro-machined piezoresistive accelerometer. The result is quite simple in terms of the variation of output voltage at different accelerations and temperatures. On the other hand, the optimization targets have not been mentioned.

In 2006, Pramanik C. et al. [5] presented a design optimization of high performance conventional silicon-based pressure sensors on flat diaphragms for low-pressure biomedical applications. It was achieved by optimizing the doping concentration and the geometry of the piezoresistors. A new figure of merit called the performance factor (PF) is defined as the sensitivity $(\mathrm{S})$ and signal-to-noise (SNR) ratio of the sensor to the temperature coefficient of piezoresistance (TCPR). PF has been introduced as a quantitative index of the overall performance of the pressure sensor for low-range biomedical applications.

In 2002, Rodjegard H. et al. [6] presented analytical models for three axis accelerometers based on four seismic masses. The models make it possible to better understand and predict the behavior of these accelerometers. Cross-axis sensitivity, resolution, frequency response and direction dependence are investigated for a variety of sensing element structures and readout methods. With the maximum sensitivity direction of the individual sensing elements inclined at $35.3^{\circ}$ with respect to the chip surface, the properties become direction independent, i.e. identical resolution and frequency response in all directions.

In the present work, a miniaturized piezoresistive three-degree of freedom accelerometer with adjustable resolutions is developed using MEMS technology. A flexure configuration is proposed in order to achieve requirements of small cross-axial acceleration, high sensitivity, and small resolution. The overall chip dimension is $1.5 \times 1.5 \times 0.5 \mathrm{~mm}^{3}$ $(L \times W \times T)$. Twelve piezoresistors are diffused on the surface of the beam structure. Three simple Wheatstone bridges are formed directly on this sensor by interconnecting these piezoresistors to sense three components of acceleration independently. A completed simulation and analysis is performed by using ANSYS simulation environment.

To obtain a high resolution, the sensitivity has to be maximized and the noise should be minimized. They both can be solved by reducing the resonance frequency. However, the practice fabrication parameters could not overcome the limitation values (the beam structure can be easily cracked while increasing the length or reducing the thickness and width of the beam). Thus, the sensitivity of the sensor was first optimized by suitably placing the piezoresistor on the surface of the four beams. After that, the noise can be reduced by narrowing the bandwidth of the accelerometer by using an analogue low pass filter in the readout circuit. 


\section{THE PROPOSED 3-DOF ACCELEROMETER WITH ADJUSTABLE AXIAL RESOLUTIONS}

The three-degree-of-freedom accelerometer always requires a small cross-axial acceleration, high and linear sensitivity, and small resolutions. Therefore, a flexure configuration, as shown in Fig.1, is proposed in order to meet these critical characteristics [7].

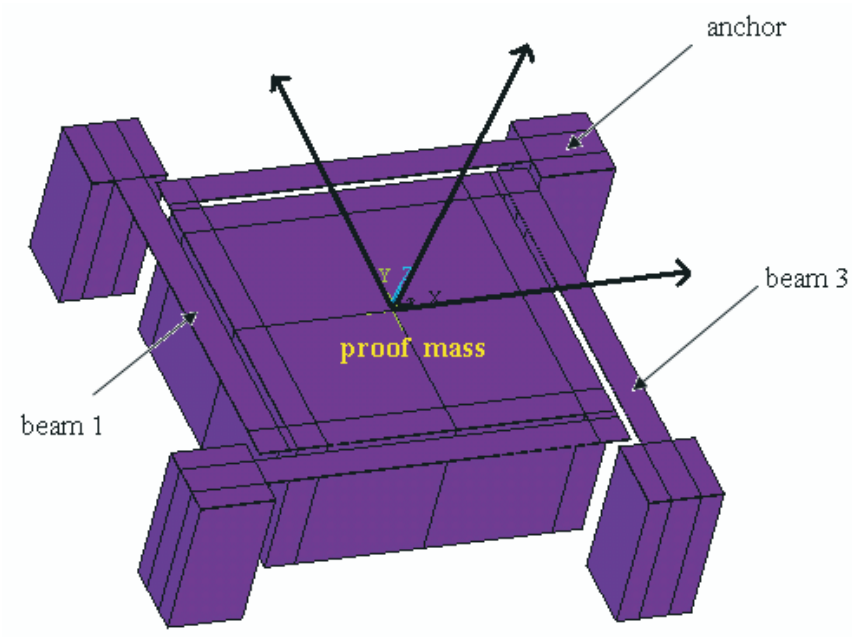

Fig. 1. 3D model of the 3-DOF piezoresistive accelerometer

An external acceleration results in a force being exerted on the mass. This force results in deflection of the proof mass. The acceleration component $(A Z)$ causes the mass to move vertically up and down. The second type of motion is caused by the $X$ or $Y$ component of transversal accelerations. The deflection of the proof mass causes stress variations on the four beam surfaces. This phenomenon in turn provokes resistance variations in the piezoresistors embedded on the surface of the beam structure.

Such variations are converted into electrical signals by using three Wheatstone bridge circuits. They are simple and it is possible to integrate electronic circuitry directly on the sensor chip for signal amplification and temperature compensation. They were built by interconnecting twelve p-type piezoresistors $[8,9]$. These p-type piezoresistors were chosen to diffuse on the surface of these four beams because they can provide the maximal resistance variations.

These piezoresistors were aligned with the crystal directions $<110>$ and $<1 \overline{1} 0>$ of n-type silicon (100). These designed piezoresistors were identified and fabricated by the diffusion method.

The piezoresistance effect is known to be caused by the anisotropic characteristics of the energy resolution in the crystal space [8]. In the silicon material, there are only three independent piezoresistive coefficients $\pi_{11}, \pi_{12}$ and $\pi_{44}$. The longitudinal piezoresistance coefficient $\pi_{l}$ is defined in the case the stress parallels with the direction of the electric filed and current density. Similarly, the transverse piezoresistance coefficient $\pi_{t}$ is defined in the 
case the stress is perpendicular with the direction of the electric filed and current density. In directions $\left\langle 110>\right.$ and $\left\langle 1 \overline{1} 0>\right.$ of n-type silicon (100), $\pi_{l}, \pi_{t}$ coefficients is calculated through three independent coefficients $\pi_{11}, \pi_{12}$ and $\pi_{44}$ as the following equation [9]:

$$
\begin{aligned}
& \pi_{l}=\frac{1}{2}\left(\pi_{11}+\pi_{12}+\pi_{44}\right) \\
& \pi_{t}=\frac{1}{2}\left(\pi_{11}+\pi_{12}-\pi_{44}\right)
\end{aligned}
$$

From simulation, two normal stresses are rather smaller as compared to the longitudinal stress $\sigma_{l}$. This phenomenon will affect to the sensitivity of the sensor. To eliminate this effect, we should avoid placing piezoresistors near the fixed end and the start of the beam. Thus, the relative change of resistance can be calculated through the normal stress by the following equation:

$$
\frac{\Delta R}{R} \approx \pi_{l} \sigma_{l}
$$

The mechanical sensitivities of each components of the acceleration can be respectively expressed as:

$$
S_{\text {stress }}^{i}=\frac{\sigma^{i}}{a_{i}}, \quad i=X, Y, Z
$$

where $S_{\text {stress }}^{i}$ is the mechanical sensitivity and $\sigma^{i}$ is the longitudinal stress induced by the acceleration $i^{\text {th }}$ component $a_{i}$. The electronics sensitivity can be given by:

$$
S_{i}=\frac{V_{\text {out }}}{a_{i}}=\frac{\Delta R}{R} V_{\text {in }}=\pi_{l} S_{\text {stress }}^{i} V_{\text {in }}
$$

where $S_{i}$ and $V_{\text {out }}$ are the sensitivity to the $i^{\text {th }}$ acceleration component and output voltage, respectively. The longitudinal stress $\sigma^{i}$ in Eq. (3) obtained from the stress analysis by using ANSYS simulation. This value is stress at the center point of piezoresistors and on the beam surface.

From Eq. (4), it is obvious that the sensitivities in three axes can be optimized if the twelve piezoresistors are diffused at specific locations that can lead to maximize the longitudinal stresses $\sigma^{i}$.

The proof mass $m$ moves from its neutral position relative to the frame when the frame starts to accelerate. For a given acceleration $a$, the proof mass displacement $x$ is determined by the mechanical suspension $k$ and the damping $b$.

$$
\frac{d^{2} x}{d t^{2}}+\frac{b}{m} \frac{d x}{d t}+\frac{k}{m} x=a(t)
$$

This equation can be rewritten:

$$
\frac{d^{2} x}{d t^{2}}+2 \xi \omega_{n} \frac{d x}{d t}+\omega_{n}^{2} x=a(t)
$$

where $\omega_{n}=\sqrt{\frac{k}{m}}$ is the natural resonance frequency, and $\xi=\frac{b}{2 m \omega_{n}}$ is the damping factor. The transfer function can be obtained as:

$$
\frac{X(s)}{A(s)}=\frac{1}{s^{2}+2 \xi \omega_{n} s+\omega_{n}^{2}}
$$


It can be seen that an open loop accelerometer is equivalent to a "mechanical" low pass filter. The natural resonance frequency is an important parameter in an open loop accelerometer design. From the definition of resonance frequency, the accelerometer with a large mass can achieve the high sensitivity by reducing the resonance frequency. In practice, it is designed to satisfy the requirements on the sensitivity and the bandwidth. The bandwidth is defined as:

$$
\Delta \omega=\omega_{2}-\omega_{1}, \quad \omega_{2}>\omega_{1}
$$

where $\omega_{1}, \omega_{2}$ are the two cut-off frequencies (or half-power frequencies) at which the amplitude response of transfer function is 0.707 . The bandwidth can be also rewritten by the Eq. (9) [10]:

$$
\Delta \omega=\omega_{2}-\omega_{1}=2 \xi \omega_{n}
$$

It can be seen that the bandwidth is also proportional to the damping ratio. The bandwidth can be often chosen at the value of $20 \%$ of resonance frequency to ensure the stability of sensor (in the free air).

Resolution is defined as the noise divided by the sensitivity. It is observed that optimization of the resolution has been achieved by increasing the sensitivity and reducing the noise. There are two typical noise sources existing in all piezoresistive sensors, including the Johnson and flicker noise $[11,12]$. The noises depend on the bandwidth of the sensor, the temperature, the geometry of the piezoresistor, the doping concentration and also the thickness of the beam.

Johnson noise (thermal noise) is the electronic noise generated by the thermal agitation of the charge carriers inside an electrical conductor when applying an arbitrary voltage. The power spectral density (PSD) of thermal noise is nearly constant throughout the frequency spectrum. This means that Johnson noise can be assumed to be White noise.

In fact, it is hard to observe this noise in a realistic accelerometer because electrical noise in the measurement circuit is often larger. The root mean square voltage of equivalent acceleration noise in each piezoresistor is:

$$
V_{i}^{\text {Johnson }}=\sqrt{4 k_{B} T B_{i} R}, \quad i=X, Y, Z
$$

where $k_{B}=1.38 \times 10^{-23} \mathrm{~J} / \mathrm{K}$ is the Boltzmann's constant, $T$ is the temperature in resistors, $R$ is the resistance value of the piezoresistor, and $B$ is the measured bandwidth. The bandwidth can be determined by many parameters such as the sampling frequency of the analogue filtering, the resonance frequency of the mechanical structure, or losses in the wires, etc.

Resolution is defined as the noise divided by the sensitivity:

$$
R_{i}=\frac{V_{i}^{\text {noise }}}{S_{i}}, \quad i=X, Y, Z
$$

It can be seen that the resolution can be minimized by reducing noise and maximizing the sensitivity. The sensitivity can not be quite large due to fabrication constraints. However, the noise can be reduced by constraining the bandwidth of the accelerometer by integrating a low pass filter at the circuit interface which has a two-order transform 
function as:

$$
C(s)=\frac{1}{s^{2}+2 \xi_{c} \omega_{c n} s+\omega_{c n}^{2}}
$$

where $a_{1}=2 \xi_{c} \omega_{c n}$ and $a_{2}=\omega_{c n}^{2}$ are two coefficients of the analogue low pass filter.

The constraint bandwidth is adjusted by using a variable resistor to adjust $a_{1}$ and $a_{2}$.

The combined system has the transform function given by:

$$
H(s)=\frac{1}{s^{2}+2 \xi \omega_{n} s+\omega_{n}^{2}} \frac{1}{s^{2}+2 \xi_{c} \omega_{c n} s+\omega_{c n}^{2}}
$$

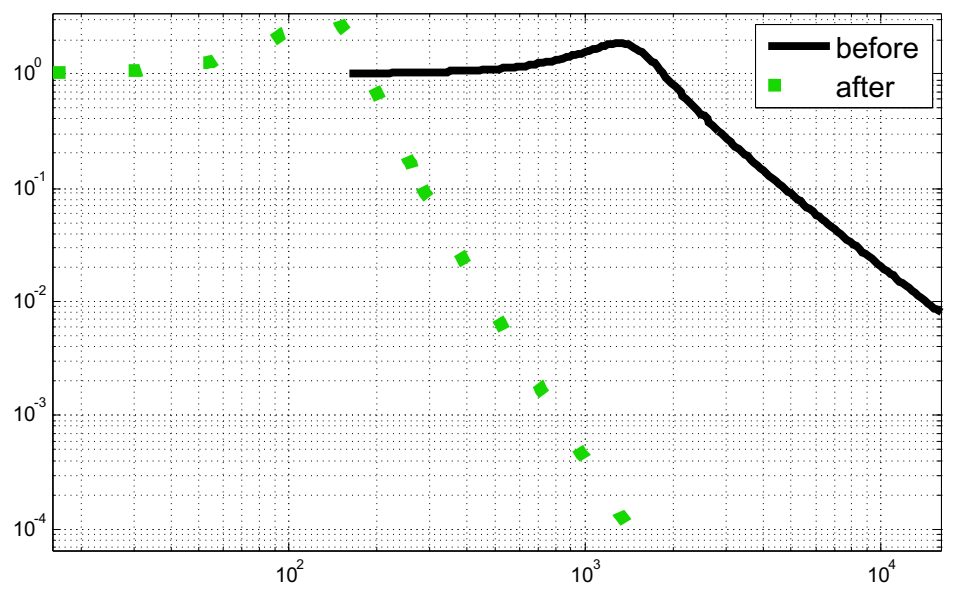

Fig. 2. Frequency responses of the accelerometer before and after bandwidth constraint

Fig. 2 shows the illustration of a low pass filter to reduce the bandwidth $B_{i}$ of the accelerometer. From the equations (8) and (9), the resolution $R_{i}$ can be three times improved. Note that this method does not change the sensitivity; thus, ensures the stability of the accelerometer.

\section{DESIGN AND SIMULATION USING ANSYS}

The finite element method (FEM) is applied to perform analyses of the stress distribution in the flexure beams. Based on the stress distribution, piezoresistors are placed to eliminate the cross-axis sensitivities and maximize the sensitivities to three components of the acceleration. The finite element model of the sensing chip was analyzed by using ANSYS.

Fig. 3 shows the mesh generation for the analysis and Fig. 4 shows the stress distribution in the longitudinal and transverse directions of the first beam caused by the acceleration $A X$. Clearly, the stress distribution in the direction along the beam (the longitudinal stress) is much larger than that of the transverse one.

The parameter of the structure brought to the Finite Element Method (FEM) process is shown in Table 1. Finite Element Analysis (FEA) based tools - although complex 


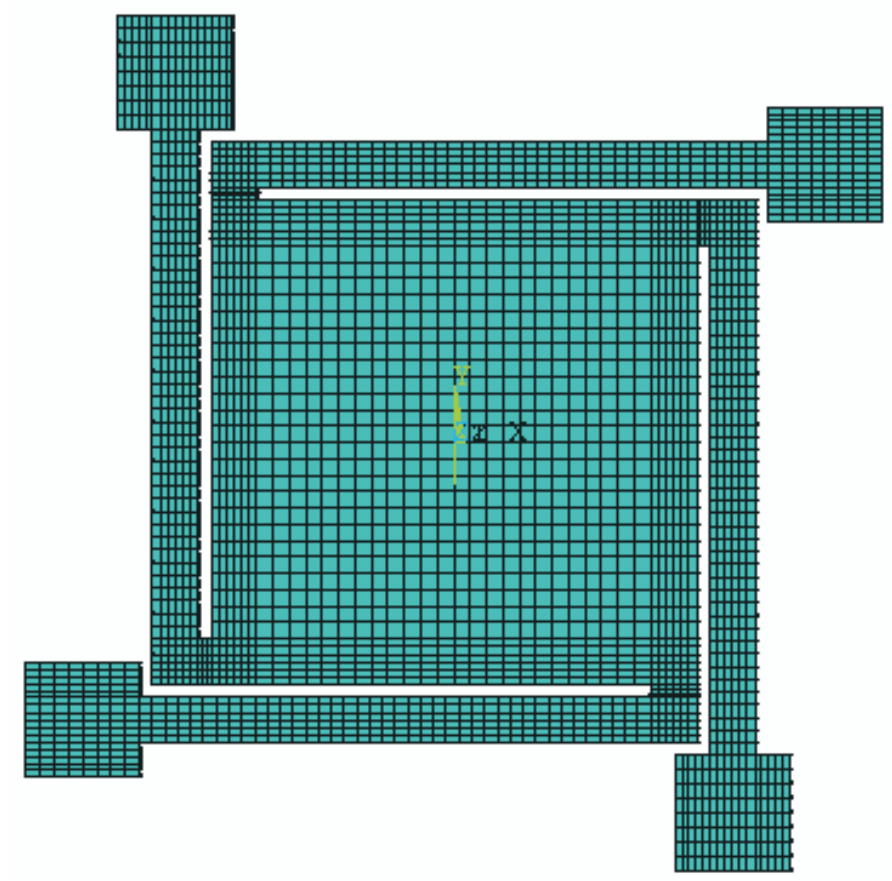

Fig. 3. The dense mesh generation of the FEM model

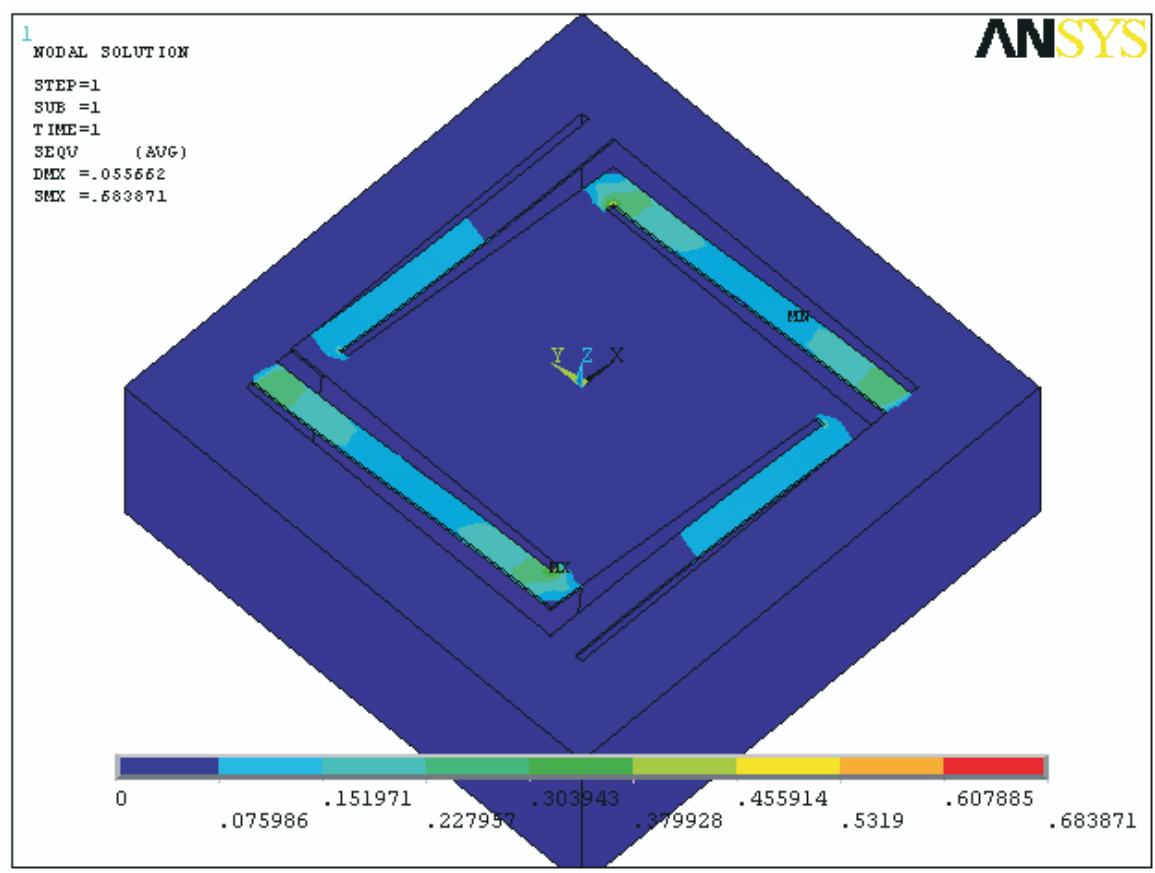

Fig. 4. The stress distribution on the beams caused by the acceleration $A X$ 
- yield more complete and precise numerical results and are especially more flexible in choosing the device geometry.

Table 1. Sensor Parameters after Manual Tuning and Synthesis Block

\begin{tabular}{|l|r|}
\hline \multicolumn{1}{|c|}{ Parameter } & \multicolumn{1}{c|}{ Size } \\
\hline Mass & $845 \times 845 \times 400 \mu \mathrm{m}^{3}$ \\
\hline Beam & $975 \times 80 \times 10 \mu \mathrm{m}^{3}$ \\
\hline Die size & $1.5 \times 1.5 \times 0.5 \mathrm{~mm}^{3}$ \\
\hline Outer frame width & $200 \mu \mathrm{m}$ \\
\hline
\end{tabular}

The most important aspect of this design process which requires FEA is the analysis of the stress distribution in the flexure beams. Based on this distribution, piezoresistors are positioned to eliminate the cross-axis sensitivities and maximize the sensitivities to the three acceleration components. Stress analysis is then performed to determine the stress distribution in the beams (Fig. 4). The sensing principle of the sensor is based on the characteristic of the $p$-type piezoresistor [8]. The resistance decreases when the sensor is exerted by a compressive stress and increases when it is exerted by a tensile stress.

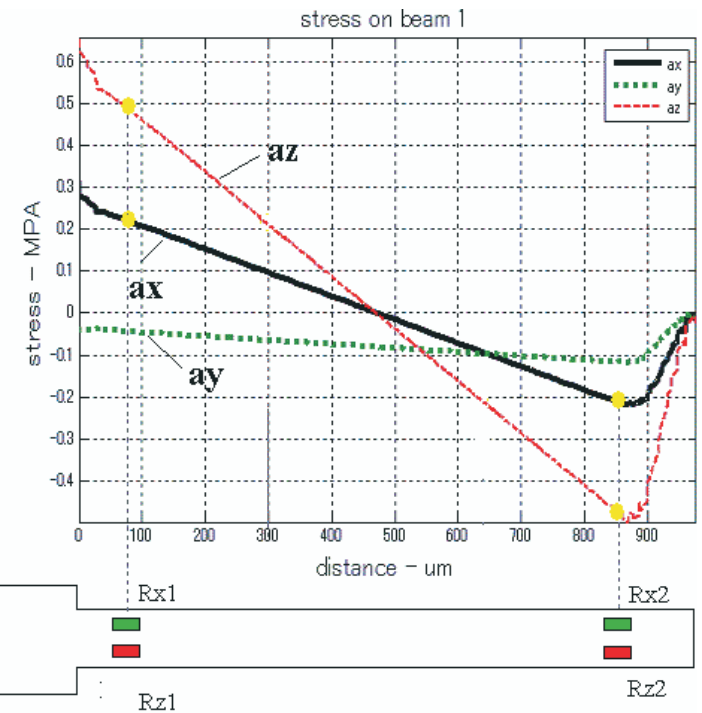

(a)

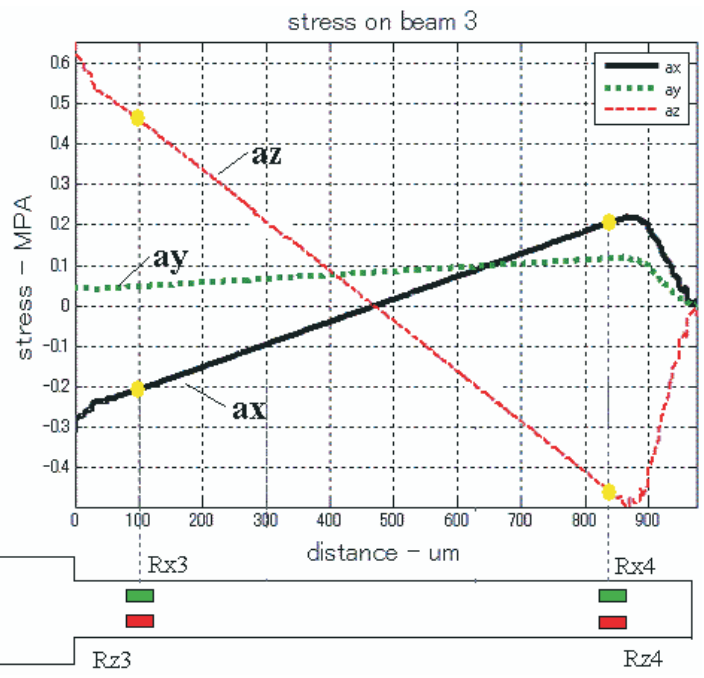

(b)

Fig. 5. Longitudinal stresses on the surface of the $1^{\text {st }}(5 . \mathrm{a})$ and the $3^{\text {rd }}(5 . \mathrm{b})$ beams due to the $1 g$ acceleration

Fig. 5 shows the stress analysis results along the $1^{\text {st }}$ and the $3^{r d}$ beams when the sensor is submitted to acceleration in three directions $(X, Y$ and $Z)$. From this figure, the optional locations can be pointed out to diffuse the piezoresistors in order to sense accelerations $A X$ and $A Z$ with very small cross-talk. To get the maximum stress corresponding 
to three cases of applying acceleration, the locations of piezoresistors are also illustrated. By the same token, acceleration $A Y$ can be sensed via four piezoresistors on the $2^{\text {nd }}$ and the $4^{\text {th }}$ beams.

Table 2 summarizes the increase $(+)$, decrease $(-)$, or invariance $(0)$ in resistance of piezoresistors due to application of accelerations $A X, A Y$, and $A Z$. The identical piezoresistors are diffused on the beam surfaces to form three Wheatstone bridges [3]. They are then connected to three corresponding low pass filters that have been discussed in the Section 2.

Table 2. Resistance Values Changes with Three Components of Acceleration

\begin{tabular}{|c|c|c|c|c|c|c|c|c|c|c|c|c|}
\hline Acc & $\boldsymbol{R} \boldsymbol{z}_{1}$ & $\boldsymbol{R} \boldsymbol{z}_{2}$ & $\boldsymbol{R} \boldsymbol{z}_{3}$ & $\boldsymbol{R} \boldsymbol{z}_{4}$ & $\boldsymbol{R} \boldsymbol{y}_{1}$ & $\boldsymbol{R} \boldsymbol{y}_{2}$ & $\boldsymbol{R} \boldsymbol{y}_{3}$ & $\boldsymbol{R} \boldsymbol{y}_{4}$ & $\boldsymbol{R} \boldsymbol{x}_{1}$ & $\boldsymbol{R} \boldsymbol{x}_{2}$ & $\boldsymbol{R} \boldsymbol{x}_{3}$ & $\boldsymbol{R} \boldsymbol{x}_{4}$ \\
\hline$A z$ & + & - & + & - & - & - & - & - & - & - & - & - \\
\hline$A y$ & - & 0 & + & 0 & - & + & - & + & 0 & + & + & 0 \\
\hline$A x$ & - & - & + & + & 0 & + & + & 0 & - & + & - & + \\
\hline
\end{tabular}

By using ANSYS simulation tool, we also obtained the first three resonance frequencies (shown in Table 3). The performance of the accelerometer is summarized in Table 4.

Table 3. First Three resonance Frequencies of the Structure

\begin{tabular}{|c|c|c|}
\cline { 2 - 3 } \multicolumn{1}{c|}{} & Resonant frequency (kHz) & Recommended bandwidth (kHz) \\
\hline AZ & 1.51 & 0.3 \\
\hline AX, AY & 98.5 & 20 \\
\hline
\end{tabular}

With the bandwidth adjustment obtained by the combination of a "mechanical" filter with a electronics filter illustrated in Fig. 2, the resolutions have been improved three times compared to the before ones. In practice, the quantity of the improved factor can be changed by the variable resistor of the electronic filter, depending on applications.

Table 4. Performance parameters of the sensor

\begin{tabular}{|c|c|c|c|c|}
\hline Acc & $\begin{array}{c}\text { Sensitivity } \\
(\mathbf{m V} / \mathbf{V} / \mathbf{g})\end{array}$ & $\begin{array}{c}\text { Johnson noise per 1 } \\
\text { piezoresistor }(\boldsymbol{\mu} \mathbf{V})\end{array}$ & $\begin{array}{c}\text { Resolution (mg) } \\
\text { before improvements }\end{array}$ & $\begin{array}{c}\text { Resolution (mg) } \\
\text { after improvements }\end{array}$ \\
\hline$A Z$ & 0.336 & 0.415 & 0.495 & 0.151 \\
\hline$A X, A Y$ & 0.152 & 0.513 & 1.353 & 0.414 \\
\hline
\end{tabular}

\section{CONCLUSION}

This paper presents design and simulation of the 3-DOF MEMS based on the accelerometer with adjustable resolution. The piezoresistive effect is used as sensing principle of the sensor. The most important aspect of the FEA in this design process is the analysis of the stress distribution in the four flexure beams. The stress analysis is performed in order to determine positions of the doped piezoresistors on these beams. The miniature 3-DOF accelerometer with adjustable resolution is expected to be applied in various applications such as biomedicine, robotics, and navigation systems. 


\section{ACKNOWLEDGMENT}

This work is partly supported by the project QG-B-2011. The authors would like to acknowledge the Advanced Micro/Nano System Technology Research Center, Ritsumeikan University, Japan for their supports, and suggestions.

\section{REFERENCES}

[1] Roylance L. M., Angell J. B, A batch-fabricated silicon accelerometer, IEEE Transactions on Electron Devices, 26(12) (1979) 1911 - 1917.

[2] Ramos J., Sensitivity enhancement in lateral capacitive accelerometers by structure width optimsation, Electronics Letters, 33 (1997) 384 - 386.

[3] Harkey J. A., Kenny T. W., $1 / f$ Noise Considerations For The Design And Process Optimization Of Piezoresistive Cantilevers, Journal of Microelectromechanical Systems, 9 (2000) 226 235.

[4] Sankar A. R., Saini V., Das S., Kal S., Temperature drift analysis in silicon micromachined piezoresistive accelerometer, Proceedings of the IEEE INDICON 2004, India, (2004) 561 - 563.

[5] Pramanik C., Saha H. and Gangopadhyay U., Design optimization of a high performance silicon MEMS piezoresistive pressure sensor for biomedical applications, J. Micromech. Microeng., 16 (2006) 2060 - 2066.

[6] Rodjegard H., Andersson G., Design optimization of three-axis accelerometers based on four seismic masses, Proceedings of IEEE Sensors, 2 (2002) 1099 - 1104.

[7] Tan T. D., Roy S., Thuy N. P., Huynh H. T, Streamlining the Design of MEMS Devices: An Acceleration Sensor, IEEE Circuits and Systems Magazine, 8(1), (2008) 18 - 27.

[8] Yozo Kanda, Piezoresistance Effect of Silicon, Sensors and Actuators, A 28 (1981) 83 - 91.

[9] Kanda Y., Graphical representation of the piezoresistance coefficients in Si shear coefficients in plane, Japanese J. Appl. Phys., 26(7) (1987) 1031 - 1033.

[10] Stephen D. Senturia, Microsystem Design, Springer, (2001), ISBN 0-7923-7246-8.

[11] Cornelius T. Leondes el at, MEMS/NEMS Handbook Techniques and Applications, Springer Press, (2006).

[12] Spencer R. P., Fleischer B. M., Barth P. W., and Angell J. B., A theoretical study of transducer noise in piezoresistive and capacitive pressure sensors, IEEE Trans. Electron Devices, 35 (1988) $1289-1298$. 